\title{
Non-equilibrium reversible dynamics of work production in four-spin system in a magnetic field
}

\author{
E.A. Ivanchenkd* \\ Institute for Theoretical Physics, National Science Center "Kharkov Institute of Physics and Technology", \\ 1 Akademichna Str., 61108 Kharkiv, Ukraine
}

Received January 5, 2011, in final form May 25, 2011

A closed system of the equations for the local Bloch vectors and spin correlation functions is obtained by decomplexification of the Liouville-von Neumann equation for 4 magnetic particles with the exchange interaction that takes place in an arbitrary time-dependent external magnetic field. The analytical and numerical analysis of the quantum thermodynamic variables is carried out depending on separable mixed initial state and the magnetic field modulation. Under unitary evolution, non-equilibrium reversible dynamics of power production in the finite environment is investigated.

Key words: reversible dynamics, work production, spin system, magnetic field

PACS: 05.30.-d, 03.65.Aa, 44.05.+e, 05.70.Ln

\section{Introduction}

The classical thermodynamic heat engine converts heat energy into mechanical work with the help of a classical mechanical system in which some gas expands and pushes a piston in a cylinder. Such heat engine receives energy from a high-temperature reservoir. Part of energy from this reservoir is converted to mechanical work, and part of it is transferred to a low-temperature reservoir. The classical heat engine reaches its peak efficiency when it is reversible. Due to the impossibility of constructing an ideally reversible heat engine, in 1824 Carnot [1] offered the mathematical model for an ideal heat engine which is not only reversible, but is also cyclic. In the last decades great efforts have been made to investigate the quantum properties of the working substance, the search and practical implementation of the quantum analogue of the Carnot cycle in microsystems.

The operation of quantum heat engines that employ multi-level systems as working agents, for example harmonic oscillators, free particles in a box, three-level atoms or electrons subjected to magnetic fields, were introduced in [2 -4]. The two-level quantum systems similar to particles with spin $1 / 2$ are essential ingredients for quantum computation, but the coupled spin systems can also be used as quantum thermodynamic engines $5-10$. The quantum analogue of the Carnot cycle requires a dynamical description of the working medium, the power output and the heat transport mechanism. The spin system "working gas" in an external field has its own physical properties. These properties at weak interaction with the environment (heat baths) can, as a rule, be deformed slightly. The purpose of this paper is to predict these properties and find out what can be expected concerning the derivation of an equation for the reduced matrix of a system in contact with an environment, usually considered in the Markovian approximation.

Based on quantum mechanics, we investigate the limiting case when interactions of the working gas, consisting of 4 particles with spin l/2 in a variable magnetic field, with heat baths are to equal to zero. In the other words, a unitary time evolution is studied, and the state of the system during this evolution is interpreted using thermodynamic concepts.

The paper is organized as follows. In section 2 we introduce the model Hamiltonian. In section 3 we use the Bloch representation, in terms of the local Bloch vectors and spin correlation functions, to write down the Liouville-von Neumann equation for a density matrix for four particles with spin

*E-mail: yevgeny@kipt.kharkov.ua 
$1 / 2$ with an exchange interaction in a variable magnetic field. We describe the conservation laws which effectively supervise the numerical calculations. In section 4 we describe the local quantum thermodynamic parameters of the spin subsystems. Our numerical results are detailed in section 5 . For the separable mixed initial state we numerically investigate the quantum thermodynamics of a particle in the environment of three others depending on the modulation of an operating field and initial states. Our results are summarized and discussed in section [6] It will be numerically found that under unitary dynamics, the work production of one part of a system is compensated by absorption of work produced by the other part. Work production by a subsystem is accompanied by entropy growth and vice-versa. The entropy decreases when work is absorbed by the subsystem. Some necessary additional details for numerical results are presented in the appendix.

\section{Model Hamiltonian}

The Hamiltonian of four coupled particles $e, p, n, u$ with spin $1 / 2$ in the external ac magnetic field $\mathbf{h}=\left(h_{1}, h_{2}, h_{3}\right)$ looks like

$$
\begin{gathered}
\hat{H}=h_{i}^{\mathrm{e}} s_{i}^{\mathrm{e}}+h_{i}^{\mathrm{p}} s_{i}^{\mathrm{p}}+h_{i}^{\mathrm{n}} s_{i}^{\mathrm{n}}+h_{i}^{\mathrm{u}} s_{i}^{\mathrm{u}}+2\left(J^{\mathrm{ep}} s_{i}^{\mathrm{e}} s_{i}^{\mathrm{p}}+J^{\mathrm{en}} s_{i}^{\mathrm{e}} s_{i}^{\mathrm{n}}+J^{\mathrm{eu}} s_{i}^{\mathrm{e}} s_{i}^{\mathrm{u}}\right. \\
\left.+J^{\mathrm{pn}} s_{i}^{\mathrm{p}} s_{i}^{\mathrm{n}}+J^{\mathrm{pu}} s_{i}^{\mathrm{p}} s^{\mathrm{u}}{ }_{i}+J^{\mathrm{nu}} s_{i}^{\mathrm{n}} s^{\mathrm{u}}{ }_{i}\right)
\end{gathered}
$$

where $h_{i}^{\mathrm{e}}, h_{i}^{\mathrm{p}}, h_{i}^{\mathrm{n}}, h_{i}^{\mathrm{u}}$ are the Cartesian components of the external magnetic field in the energy units, operating on the corresponding particle ( we set the Bohr magneton $\mu_{\mathrm{B}}$ equal to 1 ); $s_{i}^{\mathrm{e}}=$ $\frac{1}{2} \sigma_{i} \otimes \sigma_{0} \otimes \sigma_{0} \otimes \sigma_{0}, s_{i}^{\mathrm{p}}=\frac{1}{2} \sigma_{0} \otimes \sigma_{i} \otimes \sigma_{0} \otimes \sigma_{0}, s_{i}^{\mathrm{n}}=\frac{1}{2} \sigma_{0} \otimes \sigma_{0} \otimes \sigma_{i} \otimes \sigma_{0}, s_{i}^{\mathrm{u}}=\frac{1}{2} \sigma_{0} \otimes \sigma_{0} \otimes \sigma_{0} \otimes \sigma_{i}$ are the matrix representation of spin operators (2.1); the Pauli matrices are

$$
\sigma_{0}=\left(\begin{array}{cc}
1 & 0 \\
0 & 1
\end{array}\right), \quad \sigma_{1}=\left(\begin{array}{cc}
0 & 1 \\
1 & 0
\end{array}\right), \quad \sigma_{2}=\left(\begin{array}{cc}
0 & -i \\
i & 0
\end{array}\right), \quad \sigma_{3}=\left(\begin{array}{cc}
1 & 0 \\
0 & -1
\end{array}\right)
$$

$\otimes$ is the symbol of direct product [11]; $J^{\mathrm{ep}}, J^{\mathrm{en}}, J^{\mathrm{eu}}, J^{\mathrm{pn}}, J^{\mathrm{pu}}, J^{\mathrm{nu}}$ are the constants of isotropic exchange interaction between spins; the summation over $e, p, n, u$ is absent.

\section{Decomplexification of the Liouville-von Neumann equation}

The Liouville-von Neumann equation for the density matrix $\rho$, describing the dynamics of four-spin system, looks like

$$
\mathrm{i} \partial_{t} \rho=[\hat{H}, \rho], \quad \rho(t=0)=\rho_{0} .
$$

Let us present the solution of the equation (3.1) as

$$
\rho=\frac{1}{16} R_{\alpha \beta \gamma \delta} \sigma_{\alpha} \otimes \sigma_{\beta} \otimes \sigma_{\gamma} \otimes \sigma_{\delta}, \quad \rho^{+}=\rho, \quad \operatorname{Tr} \rho=1, \quad R_{0000}=1
$$

Hereinafter summation is taken over the repeating Greek indices from zero up to three, and over Latin indices from one up to three. The four coherence vectors (the Bloch vectors) widely used in the magnetic resonance theory, are written as

$$
\begin{aligned}
& R_{i 000}=\operatorname{Tr} \rho \sigma_{i} \otimes \sigma_{0} \otimes \sigma_{0} \otimes \sigma_{0}, \\
& R_{0 i 00}=\operatorname{Tr} \rho \sigma_{0} \otimes \sigma_{i} \otimes \sigma_{0} \otimes \sigma_{0}, \\
& R_{00 i 0}=\operatorname{Tr} \rho \sigma_{0} \otimes \sigma_{0} \otimes \sigma_{i} \otimes \sigma_{0}, \\
& R_{000 i}=\operatorname{Tr} \rho \sigma_{0} \otimes \sigma_{0} \otimes \sigma_{0} \otimes \sigma_{i} .
\end{aligned}
$$

These vectors characterize the local properties of individual spins, whereas the other tensors describe the spin correlations. All correlation functions are in the limits

$$
-1 \leqslant R_{\alpha \beta \gamma \delta} \leqslant 1
$$


As $\mathrm{i} \partial_{t} \rho^{n}=\left[\hat{H}, \rho^{n}\right],(n=1,2,3, \ldots)$ at unitary evolution there is a enumerable number of conservation laws $\operatorname{Tr} \rho=C_{1}=1, \operatorname{Tr} \rho^{2}=C_{2}, \ldots$, where $C_{n}$ are the constants of motion, from which only the first $C_{2}, C_{3}, \ldots, C_{16}$ are algebraically independent [12]. From the conservation of purity, for which $\left(\rho^{2}\right)_{i k} \stackrel{\text { def }}{\equiv}(\rho)_{i k}$, the polynomial (square-law) invariants are obtained. The square polynomials also control the signs $R_{\alpha \beta \gamma \delta}$. The length of the generalized Bloch vector $b^{\text {epnu }}$ is conserved under unitary evolution:

$$
b^{\mathrm{epnu}}=\sqrt{R_{\alpha \beta \gamma \delta}^{2}-1}
$$

Having inserted the equation (3.2) into (3.1), multiply the equation (3.1) by all elements of the basis $\sigma_{\alpha} \otimes \sigma_{\beta} \otimes \sigma_{\gamma} \otimes \sigma_{\delta}$ in turn and take the trace for each equation to get the time derivatives of the correlation functions as $R_{\alpha \beta \gamma \delta}$

$$
\mathrm{i} \partial_{t} R_{\alpha \beta \gamma \delta}=\operatorname{Tr}[\hat{H}, \rho] \sigma_{\alpha} \otimes \sigma_{\beta} \otimes \sigma_{\gamma} \otimes \sigma_{\delta} ; \quad \alpha=(0,1,2,3), \ldots, \quad \delta=(0,1,2,3) .
$$

A detailed form of the system equation (3.6) is in the appendix. The derivation algorithm of the system equation (A1)- A15 is presented in [13. The Liouville-von Neumann equation accepts a real form in terms of the functions $R_{\alpha \beta \gamma \delta}$ as a closed system of 255 differential equations for the local Bloch vectors and spin correlation functions.

In case of equivalent particles at $\mathbf{h}^{\mathrm{e}}=\mathbf{h}^{\mathrm{p}}=\mathbf{h}^{\mathrm{n}}=\mathbf{h}^{\mathrm{u}}=\mathbf{h}, J^{\mathrm{ep}}=J^{\mathrm{en}}=J^{\mathrm{eu}}=J^{\mathrm{pn}}=J^{\mathrm{pu}}=$ $J^{\mathrm{nu}}=J$ from the equations (A1) - A4 and (A5) it follows that the square length of the total magnetization $\left(R_{q 000}+R_{0 q 00}+R_{00 q 0}+R_{000 q}\right)^{2}$ and the forms $R_{i i 00}, R_{i 0 i 0}, \ldots, R_{00 i i}$ are conserved.

In the system (A1)- A 15 , assuming, for example, $J^{\mathrm{eu}}=J^{\mathrm{pu}}=J^{\mathrm{nu}}=0$, we get a closed system of equations for the description of three-qubit dynamics [15].

The set of equations A1 - A15 with the given initial conditions has wide applications, since the magnetic field enters the form of arbitrary functions. First of all, it allows to make numerical calculations for continuous (paramagnetic resonance in a continuous mode) as well as for pulse modes (nuclear magnetic resonance). Secondly, by means of this system it is possible to investigate the entanglement dynamics of qubits in a magnetic field [14] since the entanglement measures are expressed in terms of the reduced density matrices or of populations. Thirdly, an important application of the system (A1)-(A15) is quantum approach to the Carnot cycle [1-7], where the working body is a finite spin chain.

\section{Quantum thermodynamic variables}

In the external field, the energy of a four-spin system is defined by the formula

$$
\begin{aligned}
E= & \operatorname{Tr} \hat{H}(t) \rho=\frac{1}{2}\left(h_{i}^{\mathrm{e}} R_{i 000}+h_{i}^{\mathrm{p}} R_{0 i 00}+h_{i}^{\mathrm{n}} R_{00 i 0}+h_{i}^{\mathrm{u}} R_{000 i}\right) \\
& +2\left(J^{\mathrm{ep}} R_{i i 00}+J^{\mathrm{en}} R_{i 0 i 0}+J^{\mathrm{pn}} R_{0 i i 0}+J^{\mathrm{eu}} R_{i 00 i}+J^{\mathrm{pu}} R_{0 i 0 i}+J^{\mathrm{nu}} R_{00 i i}\right) .
\end{aligned}
$$

The change of the total energy expectation value is equal to $\partial_{t} E=\partial_{t} \operatorname{Tr} \hat{H(t) \rho}=\operatorname{Tr} \partial_{t} \hat{H(t) \rho+}$ $\operatorname{Tr} \hat{H(t)} \partial_{t} \rho$. Due to the equation of motion (3.1) we have $\operatorname{Tr} \hat{H(t)} \partial_{t} \rho=-\mathrm{i} \operatorname{Tr} \hat{H(t)}[\hat{H(t)}, \rho]=0$. For an external static field, the Hamiltonian is independent of time, hence $\partial_{t} E=0$, that is the energy of the system is constant in time.

The change of work $W$ can be associated with a term where only the spectrum changes $\partial_{t} W=$

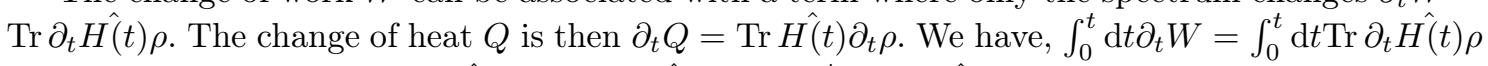
$=($ integration by parts $)=\operatorname{Tr} \hat{H(t)} \rho(t)-\operatorname{Tr} \hat{H(0)} \rho(0)-\int_{0}^{t} \mathrm{~d} t \operatorname{Tr}\left[\hat{H(t)}, \partial_{t} \rho\right]=\Delta W_{\text {sys }}$. The last integral is equal to zero due to the equation of motion. Therefore, the work production in the system is given by an expression of the form:

$$
\begin{aligned}
\Delta W_{\text {sys }} & =\operatorname{Tr} \hat{H}(t) \rho(t)-\operatorname{Tr} \hat{H}(0) \rho_{0} \\
& =\int_{0}^{t} \frac{1}{2}\left[\left(\partial_{t} h_{i}^{\mathrm{e}}\right) R_{i 000}+\left(\partial_{t} h_{i}^{\mathrm{p}}\right) R_{0 i 00}+\left(\partial_{t} h_{i}^{\mathrm{n}}\right) R_{00 i 0}+\left(\partial_{t} h_{i}^{\mathrm{u}}\right) R_{000 i}\right] \mathrm{d} t .
\end{aligned}
$$


The change in finite time $t$ of the full system energy which, in our problem, consists of the performed work and heat energy is equal to the work production since in the closed system heat energy is not produced.

The reduced density matrices describe the dynamics of subsystems (4 matrices of individual particles, 6 matrices of two particles, 4 matrices of three particles) and, for example, for a particle $e$, and the coupled particles $e p$ and $e p n$, it can be written as

$$
\begin{gathered}
\rho^{\mathrm{e}}(t)=\operatorname{Tr}_{\mathrm{pnu}} \rho=\frac{1}{2}\left(\begin{array}{cc}
1+R_{3000} & R_{1000}-\mathrm{i} R_{2000} \\
R_{1000}+\mathrm{i} R_{2000}(t) & 1-R_{3000}
\end{array}\right), \\
\rho^{\mathrm{pn}}=\frac{1}{4} R_{0 \beta \gamma 0} \sigma_{\beta} \otimes \sigma_{\gamma}, \quad \rho^{\mathrm{epn}}=\frac{1}{8} R_{\alpha \beta \gamma 0} \sigma_{\alpha} \otimes \sigma_{\beta} \otimes \sigma_{\gamma} .
\end{gathered}
$$

The matrices (4.3a) - 4.3b are determined by the system solution (A1)- A15), since the equations for the reduced matrices are not closed. From the system (A1)-A15) it follows that the spin flip probabilities of $p, n$ from their initial state are equal to

$$
P^{\mathrm{p}}=\frac{1-R_{0300}}{2}, \quad P^{\mathrm{n}}=\frac{1-R_{0030}}{2} .
$$

In the longitudinal field $\mathbf{h}=\left(0,0, h_{3}\right)$ with equal coupling constants $J$ on the equations (A1)(A4) it follows that

$$
\partial_{t} m_{q}=\varepsilon_{3 s q} h_{3} m_{s},
$$

where $m_{q}=R_{q 000}+R_{0 q 00}+R_{00 q 0}+R_{000 q}$ and $m_{3}$ is the invariant of motion. The system of equations for $m_{1}, m_{2}$ has zero solutions $m_{1}=0, m_{2}=0$ for the initial condition (A16). We believe that transverse coherences $R_{q 000}=R_{0 q 00}=R_{00 q 0}=R_{000 q}=0$ for $q=1,2$ because the numerical solution confirms that each term in $m_{1}, m_{2}$ is equal to zero during unitary evolution, that is the reduced matrix

$$
\rho^{\mathrm{e}}=\frac{1}{2}\left(\begin{array}{cc}
1+R_{3000} & 0 \\
0 & 1-R_{3000}
\end{array}\right)
$$

is diagonal. In this case it is possible to correctly define the local dynamic temperature. The local or dynamic temperature for a two-level system can be defined according to the basic meaning of a thermal state [9, 16]:

$$
T^{\mathrm{e}}(t)=-\frac{\Omega(t)}{k_{\mathrm{B}} \ln \left(p_{1} / p_{0}\right)},
$$

where $\Omega(t)$ is the transition frequency in the $e$ two-level system, equal to $h_{3}$. We assume that the interaction strength $\mathrm{J}$ between the particles is small in comparison to the energy level spacing. In this case, the interaction will not significantly alter the instantaneous energy eigenvalues of the system and hence we can meaningfully define the temperature of individual particles, since each will remain in the instantaneous thermal form (4.6), with the energy level spacing being the same as in the absence of interaction. Further we set the Bolzmann constant $k_{\mathrm{B}}$ equal to 1 . Therefore, all temperatures are in energy units. It is necessary to note a generalized approach to temperature, work and heat without weak coupling approximation between the particles [17].

The $e$-spin entropy is equal to

$$
S^{\mathrm{e}}(t)=-\operatorname{Tr} \rho^{\mathrm{e}} \ln \rho^{\mathrm{e}}=-p_{0} \ln p_{0}-p_{1} \ln p_{1},
$$

where the local populations are equal to

$$
p_{0}=\frac{1-R_{3000}}{2}, \quad p_{1}=\frac{1+R_{3000}}{2} .
$$

As the system is isolated, its entropy as a whole is constant. Hence, the system is reversible. In the reversible system, the subsystems should be reversible, that is, there exist entropy fluxes between 
subsystems if the initial state is non-equilibrium. The entropy rate $\partial_{t} S^{\mathrm{e}}$ of the $e$-spin due to the $p, n, u$ environment is defined by the formula:

$$
\partial_{t} S^{\mathrm{e}}=\frac{\partial_{t} R_{3000}}{2} \ln \frac{1-R_{3000}}{1+R_{3000}} .
$$

The stated (4.6), (4.7) and (4.9) concern each particle.

The calculation of the work carried out by subsystems is done in the papers [9] by means of $S T$ diagrams. It is shown numerically that the entropy $S$ and temperature $T$ are dependent thermodynamic variables. For a closed trajectory in the $S T$ plane, the change of the total energy $\Delta W_{\text {sys }}$ is equal to zero and consequently the area captured by the closed curve in the $S T$ plane determines the work during a reversible cycle

$$
\Delta W=-\oint T \mathrm{~d} S=-\int_{0}^{t_{\mathrm{c}}} T(t) \partial_{t} S \mathrm{~d} t,
$$

where $t_{\mathrm{c}}$ is the duration of a cycle, and the sign is defined according to the rule saying that if, at a path tracing clockwise, the area is situated on the right it obtains a minus sign (heat pump). The spin system is isolated and consequently, the Carnot cycles can only refer to sub-systems of the 4-spin system. We use (4.10) for $e, p, n, u$ particles.

The energy of the coupled particle $e$ in a magnetic field $h_{3}$ in an environment of three others is equal to $\frac{1}{2} h_{3} R_{3000}$. We use the formula $\partial_{t}\left(\frac{1}{2} h_{3} R_{3000}\right)=\frac{1}{2}\left(\partial_{t} h_{3}\right) R_{3000}+\frac{1}{2} h_{3} \partial_{t} R_{3000}$. The work production (i.e., the heat production) by spin $e$ during a cycle is equal to $\int_{0}^{t_{\mathrm{c}}} \frac{1}{2}\left(\partial_{t} h_{3}\right) R_{3000} \mathrm{~d} t$ $\left(\int_{0}^{t_{\mathrm{c}}} \frac{1}{2} h_{3} \partial_{t} R_{3000} \mathrm{~d} t\right)$. During that cycle the energy change of the particle $e \int_{0}^{t_{\mathrm{c}}} \partial_{t}\left(\frac{1}{2} h_{3} R_{3000}\right) \mathrm{d} t$ is equal to zero, hence

$$
\int_{0}^{t_{\mathrm{c}}} \frac{1}{2}\left(\partial_{t} h_{3}\right) R_{3000} \mathrm{~d} t=-\int_{0}^{t_{\mathrm{c}}} \frac{1}{2} h_{3} \partial_{t} R_{3000} \mathrm{~d} t .
$$

Having inserted the equations (4.6), (4.9) into (4.10) we conclude that

$$
\Delta W^{\mathrm{e}}=-\oint T^{\mathrm{e}} \mathrm{d} S^{\mathrm{e}}=\int_{0}^{t_{\mathrm{c}}} \frac{1}{2}\left(\partial_{t} h_{3}\right) R_{3000} \mathrm{~d} t .
$$

Thus, the definitions of temperature (4.6), entropy (4.7) and work (4.10) are coordinated with the work/heat production for parameters for which the $S T$ plots are closed. It also concerns $p, n, u$ particles.

The Klein-von Neumann inequality looks like

$$
-\operatorname{Tr} \rho \ln \rho \leqslant-\sum_{i=1}^{m} \rho_{i i} \ln \rho_{i i} \leqslant \ln m,
$$

where $-\sum_{i=1}^{m} \rho_{i i} \ln \rho_{i i}$ is the diagonal entropy, $d, m$ is the number of system states. For the initial diagonal state, the diagonal entropy possesses the property $d(0) \leqslant d(t)=-\sum_{i=1}^{m} \rho_{i i} \ln \rho_{i i}[18$ ].

The dynamics of a purity measure $P=\operatorname{Tr} \rho^{2}$ is connected with the dynamics of entropy $S=-\operatorname{Tr} \rho \ln \rho$ as follows. If the entropy is equal to zero, the system is in a pure state. At the maximum entropy, the system is in the maximum mixed state. The purity $P$ has the maximum value 1 for a pure state and the minimum value in the mixed state, equal to $1 / m$, where $m$ is the number of system states. The subsystem purity is expressed in terms of the square length of the local or generalized Bloch vector (3.5): the purity for the subsystems pn and epnu is equal to

$$
P^{\mathrm{np}}=\frac{1}{4}\left(1+b^{\mathrm{np} 2}\right), \quad P^{\mathrm{epnu}}=\frac{1}{16}\left(1+b^{\mathrm{epnu} 2}\right)
$$


accordingly, where $b^{n p}=\sqrt{\sum_{i, j=1}^{3} R_{0 i j 0}^{2}}$ is the length of the generalized Bloch vector of the $p n$ system.

Let us define the entanglement measure $p$ and $n$ for spins according to [19] on system solutions having entered the two-particle entanglement tensor:

$$
m_{0 i j 0}=R_{0 i j 0}-R_{0 i 00} R_{00 j 0}
$$

The tensor $m_{0 i j 0}$ is equal to zero when the two-particle correlation function $R_{0 i j 0}$ is factorized in terms of the local Bloch vectors (3.3a - 3.3d and thus the matrix will be separable, i.e., $\rho^{\mathrm{pn}}=$ $\rho^{\mathrm{p}} \otimes \rho^{\mathrm{n}}$. By means of this tensor we shall define a measure of the two-particle entanglement in the pn subsystem

$$
m_{\mathrm{SM}}^{\mathrm{pn}}=\sqrt{\frac{1}{3} \sum_{i, j=1}^{3} m_{0 i j 0}^{2}} .
$$

This measure is equal to zero for a separable state and it is equal to 1 for the Greenberger-HorneZeilinger maximum entangled state. This measure is applicable both to the pure and mixed states (in all 6 two-particle measures).

\section{Numerical results}

The quantum thermodynamic devices are subdivided into heat pumps and heat engines depending on functional purpose. In our model, the work absorbed or done by the system arises due to the displacement of the power levels by a magnetic field [20-22], as well as it also depends on the initial state of the system.

We will consider the effect of a variable magnetic field

$$
\mathbf{h}=\left(h_{1}, h_{2}, h_{3}\right)
$$

on the dynamics of a system in the case when the fields operating on the spins $e, p, n, u$ are equal to $\mathbf{h}$, where $h_{1}=0, h_{2}=0, h_{3}=c+1.5 \sin \omega t$ ( $c$ is the static part of the magnetic field), with an external field frequency $\omega=0.04$ and with all the exchange constants equal to 0.01 . The values of magnetic field frequency are given in the energy units.

We study the behaviour of a four-spin system in terms of local thermodynamic concepts: temperature (4.6), entropy (4.7) and work (4.10) depending on the parameters of a possible nonequilibrium initial state:

(i) $e, p, u$ particles have the same temperature $T^{\mathrm{e}}(0)=T^{\mathrm{p}}(0)=T^{\mathrm{u}}(0)=0.2$, and the temperature of the $n$ particle is equal to $T^{\mathrm{n}}(0)=0.6$;

(ii) $T^{\mathrm{e}}(0)=T^{\mathrm{p}}(0)=0.2, T^{\mathrm{n}}(0)=T^{\mathrm{u}}(0)=0.25$;

(iii) $T^{\mathrm{e}}(0)=0.21, T^{\mathrm{p}}(0)=0.225, T^{\mathrm{n}}(0)=0.264, T^{\mathrm{u}}(0)=0.28$.

For the parameters (i) of the initial state (A16), let us choose the following values for our numerical results: $h_{3}^{\mathrm{e}}(0)=h_{3}^{\mathrm{p}}(0)=h_{3}^{\mathrm{n}}(0)=h_{3}^{\mathrm{u}}(0)=c$. In figure 1 (i), $\mathrm{c}=1.5$ the parametric dependences on entropy and temperature for a cycle are shown, i.e., $S T$ cycles with negative work (bold line) which are identical for $e, p, u$ spins. The spin $n$ produces some work (thin line) from the $(e, p, u)$ - environment, while each of the spins $e, p, u$ (bold line) absorbs work. The work performed by $n$ spin, is equal to the area limited by a closed curve according to the formula (4.10) and is equal to 0.1331514. The $e, p, u$ spins perform an amount of work equal to $3 \times(-0.0443838)=-0.1331514$. Thereby, the work production of the four-spin system $\Delta W_{\text {svs }}$ (4.2) is equal to zero in full compliance with the general results for the isolated system [23 27]. It confirms the use of temperature (4.6) and entropy (4.7) as effective thermodynamic characteristics. A feature of this initial state and modulation magnetic field is that these cycles are repeated without any deformation. (It is known that the system equations (A1)-A15) with periodic coefficients, according to the Floquet theory, have periodic or quasi-periodic solutions i.e. the Floquet theory does not exclude the periodic solutions as well. This depends on the set of coefficients. We have presented this set.) In other words, each cycle comes to an end returning to the same initial state. 

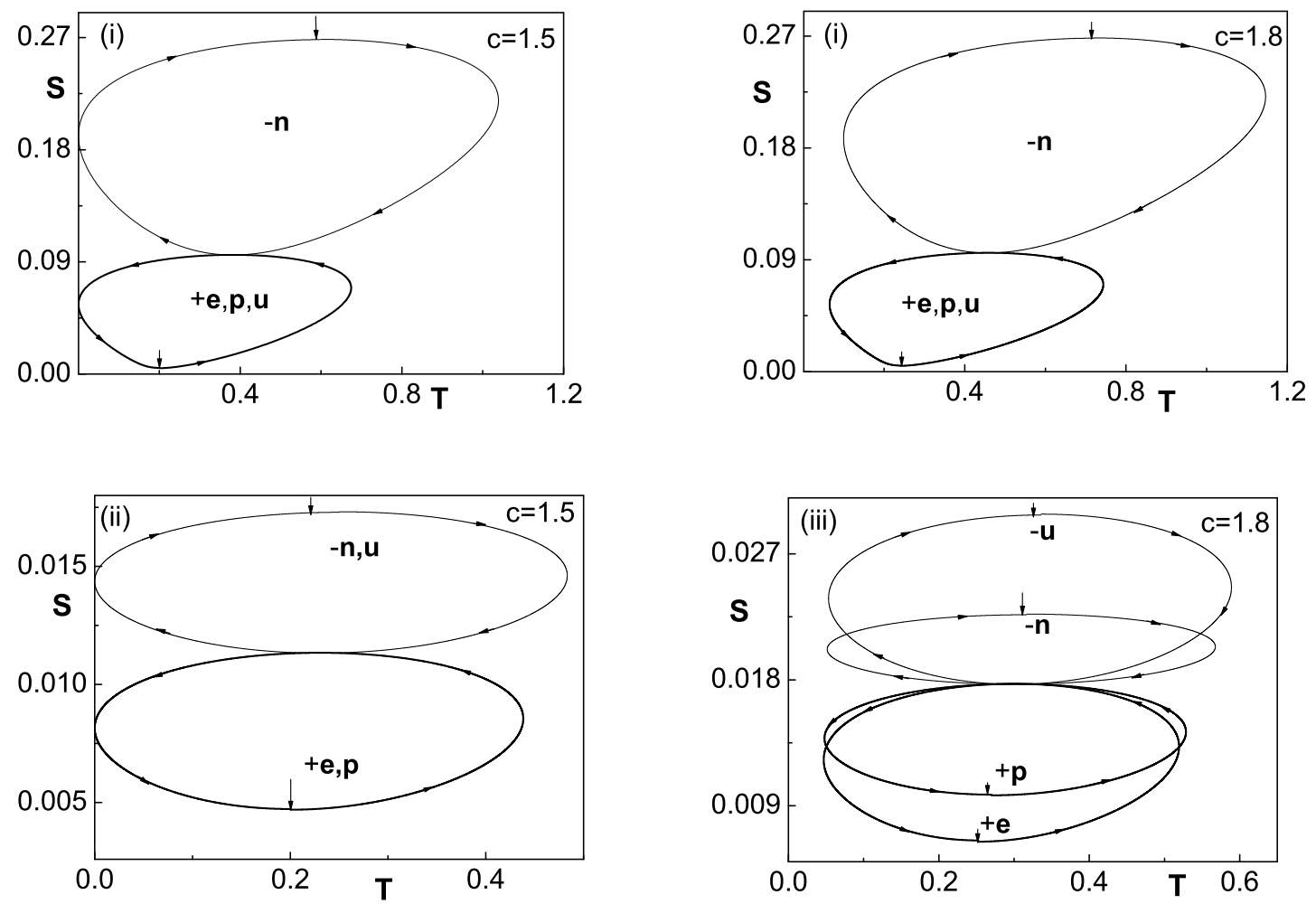

Figure 1. $S T$ diagrams for the non-equilibrium initial state with the different values of the static field $c=h_{3}^{\mathrm{e}}(0)=h_{3}^{\mathrm{p}}(0)=h_{3}^{\mathrm{n}}(0)=h_{3}^{\mathrm{u}}(0)$ and initial temperatures: (i) $T^{\mathrm{e}}(0)=T^{\mathrm{p}}(0)=$ $T^{\mathrm{u}}(0)=0.2, T^{\mathrm{n}}(0)=0.6, \quad(\mathrm{ii}) T^{\mathrm{e}}(0)=T^{\mathrm{p}}(0)=0.2, T^{\mathrm{n}}(0)=T^{\mathrm{u}}(0)=0.25, \quad($ iii $) T^{\mathrm{e}}(0)=$ $0.21, T^{\mathrm{p}}(0)=0.225, T^{\mathrm{n}}(0)=0.264, T^{\mathrm{u}}(0)=0.28$. All spins are in an identical field $\mathbf{h}$ and the exchange constants are equal to 0.01 . It is seen that the field $\mathbf{h}=(0,0, c+1.5 \sin \omega t)$ causes periodic cycles with parameters of the initial state $\omega=0.04$. The duration of a cycle is equal to $t_{\mathrm{c}}=2 \pi / \omega=157.08$. The arrows indicate the direction of circulation. The circulation direction becomes opposite for all particles after the replacement of a frequency sign $\omega$. In addition to the direction of circulation, the signs,+- specify the negative or the positive work of particles. The vertical arrows in the plots specify the return points.

The closure of the $S T$ plots does not depend on the amplitudes of the driving field (only the form and the area may change), but it critically depends on the frequency $\omega[9]$ and the module of the exchange constants, it does not depend on ferromagnetic or antiferromagnetic character of the working gas [28]. After replacement of a frequency sign $\omega$, the circulation direction becomes opposite for all particles. If the value of the temperature parameter is $T^{n}(0)>0.2$, then the particle $n$ does work, and the spins $e, p, u$ absorb work. The purity of the whole system decreases with an increase of $T^{n}(0)$, but cycle-after-cycle and periodicity remain as it is described, and the areas characterizing work increase with preservation of the algebraic sum which is equal to zero. For $T^{n}(0)<0.2$ the spins $e, p, u$ produce work, and the spin $n$ absorbs it.

If $T^{\mathrm{n}}(0)$ approaches 0.2 , that is, at the equilibrium initial state $T^{\mathrm{e}}(0)=T^{\mathrm{p}}(0)=T^{\mathrm{n}}(0)=$ $T^{\mathrm{u}}(0)=0.2$ the entropy of each subsystem is constant and the work production of each subsystem is equal to zero, since at the initial moment there is no temperature gradient in the system (passive or immovable state [29]).

For the initial state (i), $\mathrm{c}=1.8$ the work performed by the $n$ spin is equal to 0.1329435 . The $e, p, u$ spins perform an amount of work equal to $3 \times(-0.0443145)=-0.1329435$.

The numerical calculations also show that for the initial state (ii), $\mathrm{c}=1.5$ particles $e, p$ absorb 
work equal to $2(-0.002265)$, and particles $n, u$ produce the same work $2(+0.002265)[30]$.

In the case of initial state (iii) for $\mathrm{c}=1.8$ particles $e, p$ absorb work which is equal to $(-0.0041)+$ $(-0.0029)$, and particles $n, u$ produce the same work $(0.0020+0.0050)$.

We have the results of the $S T$ diagrams in figure 1 at different static parts of the magnetic field $c$ for the initial non-equilibrium states. The numerical analysis shows that the balance of work production for (i), (ii), (iii) in fact does not depend on the static magnetic field, only the local temperatures and the form of the $S T$ diagrams change.

In figure 1 it is seen that in the middle of the cycle, the non-equilibrium reversible system is converted to the quasi-equilibrium one; the entropy rates $\partial_{t} S^{i}$ change signs, the temperature rates $\partial_{t} T^{i}$ are minimum $(i=e, p, n, u)$.

We would like to indicate that in the vicinity of $t \approx 117.8$, the local temperature of all particles goes to zero as the frequencies $\Omega^{i}(t)$ for all particles go to zero. It is necessary to notice that for the minimum local temperatures, the eigenvalues of the Hamiltonian (2.1) come closer to zero, and with the growth of temperatures the eigenvalues become bigger. The transition probability of each particle (4.4) is close to 1 and makes one oscillation per cycle. The population $\rho_{1616}$ is approximately equal to 0.925 during a cycle. For the opposite sign $h_{3}^{\mathrm{e}}(0)=h_{3}^{\mathrm{p}}(0)=h_{3}^{\mathrm{n}}(0)=h_{3}^{\mathrm{u}}(0)=-1.5, \rho_{11} \approx$ 0.925 the thermodynamic characteristics do not change. In figure 1 it is clearly seen that if the initial state of a subsystem is more disordered, a subsystem absorbs/produces more (in absolute magnitude) work [29].

The cycle of each particle is determined by the direct coupling of the given particle with the others and does not depend on the coupling constants between other particles. The calculations confirm that the dependence between the energy of particles $p, n E^{\mathrm{pn}}=\left(h_{i}^{\mathrm{p}} R_{0 i 00}+h_{i}^{\mathrm{n}} R_{00 i 0}\right) / 2+$ $2 J^{\mathrm{pn}} R_{0 i i 0}$ and the entropy $S^{\mathrm{pn}}$ is also cyclic. This property is carried out by any particle pair.

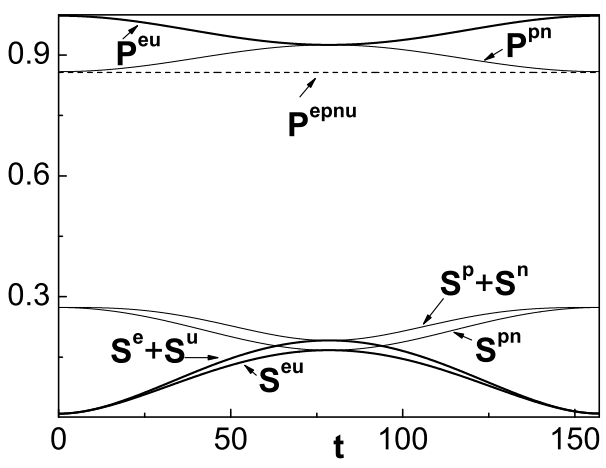

Figure 2. Time dependence on purity of all system $P^{\text {epnu }}$ and on characteristics $e u$ and $p n$ subsystems for one cycle with the parameters, corresponding to the $S T$ diagrams in figure 1 (i), $\mathrm{c}=1.5$.

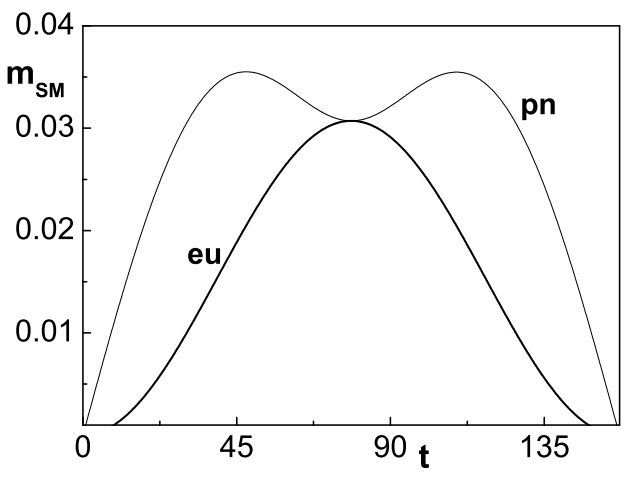

Figure 3. Time dependence on the local entanglement $m_{\mathrm{SM}}$ for subsystems $p n$ (thin line) and eu (bold line) for one cycle. The parameters correspond to the $S T$ diagrams in figure 1 (i), $\mathrm{c}=1.5$.

The results for additional quantum thermodynamic characteristics of $e u$ and $p n$ subsystems are presented in figures 2 and 3 Under unitary evolution, the global purity $P^{\text {epnu }}$ does not depend on time. The purity $P^{\mathrm{pn}}(4.12)$ has a maximum at an entropy minimum. The work production of the $e u$ subsystem is accompanied by entropy increase and purity reduction and the inverse process occurs at work absorption. It is seen that the local entanglement $m^{\mathrm{pn}}$ (4.14) has a maximum for a minimum entropy $S^{\mathrm{pn}}=-\operatorname{Tr} \rho^{\mathrm{pn}} \ln \rho^{\mathrm{pn}}$. The entanglement (4.14) between the particles is noticeable in the middle of the cycle and it is bigger between particles with opposite signs in work, and, as the calculations confirm, grows with the disorder increase in the system. This entropy is always less or equal to the sum entropies of individual spins in the $p n$ subsystem. Also, the inequality 
of Klein-von Neumann is carried out (4.11), i. e., the diagonal entropy is bigger or equal to the $p n$ subsystem entropy. For the case presented in figure 1 (i), c=1.5 the diagonal entropy $d^{\text {pn }}\left(d^{\text {eu }}\right)$ coincides with the summed entropies of the individual spins $S^{\mathrm{p}}+S^{\mathrm{n}}\left(S^{\mathrm{e}}+S^{\mathrm{u}}\right)$.

The control of calculations was carried out with the help of the invariants of the motion, described in sections 3 and 4 , and all the correlation functions have been in the limits $-1 \leqslant$ $R_{\alpha \beta \gamma \delta} \leqslant 1$.

\section{Summary}

A closed system of equations is derived for the local Bloch vectors and spin correlation functions of four two-level systems with exchange interaction, being in a time-dependent external magnetic field. The invariants of motion necessary for the control of computing have been found. Analytical and numerical analysis of thermodynamic behaviour in a four-spin weak coupling system depending on the parameters characterizing the initial non-equilibrium state and modulation of the driving field was performed. It was numerically found that under unitary dynamics, the work production of one part of the system is compensated by absorption of work produced by the other part. The work production by a subsystem is accompanied by entropy growth and vice-versa, while the entropy decreases with work absorption by the subsystem.

It is shown that in the middle of a cycle, the non-equilibrium reversible system is converted to a quasi-equilibrium one. Thermalization of non-equilibrium system of 4 spins in finite time under different initial conditions is numerically shown (figure 1). In other words, the finite non-equilibrium system in finite time generates an "attractor", i.e., an intermediate temperature is established for all particles. It was revealed that the $S T$ cycle of each particle is determined by the direct coupling of the given particle with the others and weakly depends on the coupling constants between the other spins.

It was analytically and numerically shown that the formulas for temperature (4.6) and entropy (4.7) are the effective thermodynamic characteristics for work calculation in a weakly coupled spin system by means of $S T$ diagrams in the course of unitary evolution.

The study of the "spin gas" properties is necessary for the implementation of quantum thermodynamic cycles in spin systems [31 33. Taking into account the environment, zero balance will be broken and the system will become a heat engine or a heat pump depending on the temperatures of the heat baths.

\section{Acknowledgements}

The author is grateful to Zippa A.A. for constant invaluable support and to the referees for their objective comments that improved the text in many points. 


\section{Appendix}

The detailed form of the system equation (3.6) is as follows:

$$
\begin{aligned}
& \partial_{t} R_{q 000}=\varepsilon_{i s q} h_{i}^{\mathrm{e}} R_{s 000}+\varepsilon_{s t q}\left(J^{\mathrm{ep}} R_{t s 00}+J^{\mathrm{en}} R_{t 0 s 0}+J^{\mathrm{eu}} R_{t 00 s}\right), \\
& \partial_{t} R_{0 q 00}=\varepsilon_{i s q} h_{i}^{\mathrm{p}} R_{0 s 00}+\varepsilon_{s t q}\left(J^{\mathrm{ep}} R_{s t 00}+J^{\mathrm{pn}} R_{0 t s 0}+J^{\mathrm{pu}} R_{0 t 0 s}\right) \text {, } \\
& \partial_{t} R_{00 q 0}=\varepsilon_{i s q} h_{i}^{\mathrm{n}} R_{00 s 0}+\varepsilon_{s t q}\left(J^{\mathrm{en}} R_{s 0 t 0}+J^{\mathrm{pn}} R_{0 s t 0}+J^{\mathrm{nu}} R_{00 t s}\right), \\
& \partial_{t} R_{000 q}=\varepsilon_{i s q} h_{i}^{\mathrm{u}} R_{000 s}+\varepsilon_{s t q}\left(J^{\mathrm{eu}} R_{s 00 t}+J^{\mathrm{pu}} R_{0 s 0 t}+J^{\mathrm{nu}} R_{00 s t}\right), \\
& \partial_{t} R_{q k 00}=\varepsilon_{i s q} h_{i}^{\mathrm{e}} R_{s k 00}+\varepsilon_{i s k} h_{i}^{\mathrm{p}} R_{q s 00}+J^{\mathrm{ep}} \varepsilon_{k s q}\left(R_{s 000}-R_{0 s 00}\right) \\
& +\varepsilon_{t s q}\left(J^{\mathrm{en}} R_{s k t 0}+J^{\mathrm{eu}} R_{s k 0 t}\right)+\varepsilon_{t s k}\left(J^{\mathrm{pn}} R_{q s t 0}+J^{\mathrm{pu}} R_{q s 0 t}\right), \\
& \partial_{t} R_{q 0 k 0}=\varepsilon_{i s q} h_{i}^{\mathrm{e}} R_{s 0 k 0}+\varepsilon_{i s k} h_{i}^{\mathrm{n}} R_{q 0 s 0}+J^{\mathrm{en}} \varepsilon_{k s q}\left(R_{s 000}-R_{00 s 0}\right) \\
& +\varepsilon_{t s q}\left(J^{\mathrm{ep}} R_{s t k 0}+J^{\mathrm{eu}} R_{s 0 k t}\right)+\varepsilon_{s t k}\left(J^{\mathrm{pn}} R_{q s t 0}+J^{\mathrm{nu}} R_{q 0 t s}\right), \\
& \partial_{t} R_{q 00 k}=\varepsilon_{i s q} h_{i}^{\mathrm{e}} R_{s 00 k}+\varepsilon_{i s k} h_{i}^{\mathrm{u}} R_{q 00 s}+J^{\mathrm{eu}} \varepsilon_{k s q}\left(R_{s 000}-R_{000 s}\right) \\
& +\varepsilon_{t s q}\left(J^{\mathrm{ep}} R_{s t 0 k}+J^{\mathrm{en}} R_{s 0 t k}\right)+\varepsilon_{t s k}\left(J^{\mathrm{pu}} R_{q t 0 s}+J^{\mathrm{nu}} R_{q 0 t s}\right), \\
& \partial_{t} R_{0 q k 0}=\varepsilon_{i s q} h_{i}^{\mathrm{p}} R_{0 s k 0}+\varepsilon_{i s k} h_{i}^{\mathrm{n}} R_{0 q s 0}+J^{\mathrm{pn}} \varepsilon_{k s q}\left(R_{0 s 00}-R_{00 s 0}\right) \\
& +\varepsilon_{t s q}\left(J^{\mathrm{ep}} R_{t s k 0}+J^{\mathrm{pu}} R_{0 s k t}\right)+\varepsilon_{s t k}\left(J^{\mathrm{en}} R_{s q t 0}+J^{\mathrm{nu}} R_{0 q t s}\right), \\
& \partial_{t} R_{0 q 0 k}=\varepsilon_{i s q} h_{i}^{\mathrm{p}} R_{0 s 0 k}+\varepsilon_{i s k} h_{i}^{\mathrm{u}} R_{0 q 0 s}+J^{\mathrm{pu}} \varepsilon_{k s q}\left(R_{0 s 00}-R_{000 s}\right) \\
& +\varepsilon_{s t q}\left(J^{\mathrm{ep}} R_{s t 0 k}+J^{\mathrm{pn}} R_{0 t s k}\right)+\varepsilon_{s t k}\left(J^{\mathrm{eu}} R_{s q 0 t}+J^{\mathrm{nu}} R_{0 q s t}\right), \\
& \partial_{t} R_{00 q k}=\varepsilon_{i s q} h_{i}^{\mathrm{n}} R_{00 s k}+\varepsilon_{i s k} h_{i}^{\mathrm{u}} R_{00 q s}+J^{\mathrm{nu}} \varepsilon_{k s q}\left(R_{00 s 0}-R_{000 s}\right) \\
& +\varepsilon_{s t q}\left(J^{\mathrm{en}} R_{s 0 t k}+J^{\mathrm{pn}} R_{0 s t k}\right)+\varepsilon_{s t k}\left(J^{\mathrm{eu}} R_{s 0 q t}+J^{\mathrm{pu}} R_{0 s q t}\right), \\
& \partial_{t} R_{q k l 0}=\varepsilon_{i s q} h_{i}^{\mathrm{e}} R_{s k l 0}+\varepsilon_{i s k} h_{i}^{\mathrm{p}} R_{q s l 0}+\varepsilon_{i s l} h_{i}^{\mathrm{n}} R_{q k s 0} \\
& +J^{\mathrm{ep}} \varepsilon_{k s q}\left(R_{s 0 l 0}-R_{0 s l 0}\right)+J^{\mathrm{pn}} \varepsilon_{l s k}\left(R_{q s 00}-R_{q 0 s 0}\right) \\
& +J^{\mathrm{en}} \varepsilon_{l s q}\left(R_{s k 00}-R_{0 k s 0}\right)+J^{\mathrm{eu}} \varepsilon_{s t q} R_{t k l s}+J^{\mathrm{pu}} \varepsilon_{t s k} R_{q s l t}+J^{\mathrm{nu}} \varepsilon_{t s l} R_{q k s t} \text {, } \\
& \partial_{t} R_{q k 0 l}=\varepsilon_{i s q} h_{i}^{\mathrm{e}} R_{s k 0 l}+\varepsilon_{i s k} h_{i}^{\mathrm{p}} R_{q s 0 l}+\varepsilon_{i s l} h_{i}^{\mathrm{u}} R_{q k 0 s} \\
& +J^{\mathrm{ep}} \varepsilon_{k s q}\left(R_{s 00 l}-R_{0 s 0 l}\right)+J^{\mathrm{pu}} \varepsilon_{l s k}\left(R_{q s 00}-R_{q 00 s}\right) \\
& +J^{\mathrm{eu}} \varepsilon_{l s q}\left(R_{s k 00}-R_{0 k 0 s}\right)+J^{\mathrm{en}} \varepsilon_{s t q} R_{s k t l}+J^{\mathrm{pu}} \varepsilon_{t s k} R_{q s t l}+J^{\mathrm{nu}} \varepsilon_{s t l} R_{q k s t} \text {, } \\
& \partial_{t} R_{q 0 k l}=\varepsilon_{i s q} h_{i}^{\mathrm{e}} R_{s 0 k l}+\varepsilon_{i s k} h_{i}^{\mathrm{n}} R_{q 0 s l}+\varepsilon_{i s l} h_{i}^{\mathrm{u}} R_{q 0 k s} \\
& +J^{\mathrm{en}} \varepsilon_{k s q}\left(R_{s 00 l}-R_{00 s l}\right)+J^{\mathrm{eu}} \varepsilon_{l s q}\left(R_{s o k 0}-R_{00 k s}\right) \\
& +J^{\mathrm{nu}} \varepsilon_{l s k}\left(R_{q 0 s 0}-R_{q 00 s}\right)+J^{\mathrm{ep}} \varepsilon_{t s q} R_{s t k l}+J^{\mathrm{pn}} \varepsilon_{s t k} R_{q s t l}+J^{\mathrm{pu}} \varepsilon_{s t l} R_{q s k t} \text {, } \\
& \partial_{t} R_{0 q k l}=\varepsilon_{i s q} h_{i}^{\mathrm{p}} R_{0 s k l}+\varepsilon_{i s k} h_{i}^{\mathrm{n}} R_{0 q s l}+\varepsilon_{i s l} h_{i}^{\mathrm{u}} R_{0 q k s} \\
& +J^{\mathrm{pn}} \varepsilon_{k s q}\left(R_{0 s 0 l}-R_{00 s l}\right)+J^{\mathrm{pu}} \varepsilon_{l s q}\left(R_{0 s k 0}-R_{00 k s}\right) \\
& +J^{\mathrm{nu}} \varepsilon_{l s k}\left(R_{0 q s 0}-R_{0 q 0 s}\right)+J^{\mathrm{ep}} \varepsilon_{t s q} R_{t s k l}+J^{\mathrm{en}} \varepsilon_{t s k} R_{t q s l}+J^{\mathrm{eu}} \varepsilon_{s t l} R_{s q k t} \text {, } \\
& \partial_{t} R_{q k l m}=\varepsilon_{i s q} h_{i}^{\mathrm{e}} R_{s k l m}+\varepsilon_{i s k} h_{i}^{\mathrm{p}} R_{q s l m}+\varepsilon_{i s l} h_{i}^{\mathrm{n}} R_{q k s m}+\varepsilon_{i s m} h_{i}^{\mathrm{u}} R_{q k l s} \\
& +J^{\mathrm{ep}} \varepsilon_{k s q}\left(R_{s 0 l m}-R_{0 s l m}\right)+J^{\mathrm{en}} \varepsilon_{l s q}\left(R_{s k 0 m}-R_{0 k s m}\right) \\
& +J^{\mathrm{pn}} \varepsilon_{l s k}\left(R_{q s 0 m}-R_{q 0 s m}\right)+J^{\mathrm{eu}} \varepsilon_{m s q}\left(R_{s k l 0}-R_{0 k l s}\right) \\
& +J^{\mathrm{pu}} \varepsilon_{m s k}\left(R_{q s l 0}-R_{q 0 l s}\right)+J^{\mathrm{nu}} \varepsilon_{m s l}\left(R_{q k s 0}-R_{q k 0 s}\right) .
\end{aligned}
$$

Concrete calculations were carried out for the separable mixed initial state Sep-Mix:

$$
\rho_{\mathrm{Sep}-\operatorname{Mix}}(0)=\rho^{\mathrm{e}} \otimes \rho^{\mathrm{p}} \otimes \rho^{\mathrm{n}} \otimes \rho^{\mathrm{u}},
$$


where

$$
\rho^{i}=\frac{1}{2 \cosh h_{3}^{i}(0) / 2 T^{i}(0)} \exp \left(-\frac{h_{3}^{i}(0)}{2 T^{i}(0)} \sigma_{3}\right),
$$

is the mixed state of the $i$ particle, $T^{i}(0)$ is the initial temperature, $h_{3}^{i}(0)$ is the initial field, $i=$ $(e, p, n, u)$.

\section{References}

1. Carnot S. Refléctions sur la Puissance Motrice du Feu et sur les Machines Propres à Développer Cette Puissance. Bachier, Paris, 1824.

2. Scovill H., Schulz-Dubois E.O., Phys. Rev. Lett., 1959, 2, 262; doi 10.1103/PhysRevLett.2.262,

Geusic J.E., Schulz-Dubois E.O., Scovill H., Phys. Rev., 1967, 156, 343; doi 10.1103/PhysRev.156.343.

3. Geva E., Kosloff R., J. Chem. Phys., 1992, 96, 3054; doi 10.1063/1.461951

4. Feldmann T., Geva E., Kosloff R., Salomon P., Am. J. Phys., 1996, 64, 485; doi $10.1119 / 1.18197$

5. Feldmann T., Kosloff R., Phys. Rev. E, 2004, 70, 046110; doi 10.1103/PhysRevE.70.046110.

6. Feldmann T., Kosloff R., Phys. Rev. E, 2003, 68, 016101; doi 10.1103/PhysRevE.68.016101.

7. Rezek Y., Kosloff R., New J. Phys., 2006, 8, 83; doi 10.1088/1367-2630/8/5/083 Preprint arXiv:quant$\mathrm{ph} / 0601006 \mathrm{v} 2,2006$.

8. Tonner F., Mahler G., Phys. Rev. E, 2005, 72, 066118; doi 10.1103/PhysRevE.72.066118

9. Segal D., Nitzan A., Phys. Rev. E, 2006, 73, 026109; doi 10.1103/PhysRevE.73.026109 Henrich M.J., Michel M., Mahler G., Europhys. Lett., 2006, 76, 1057; doi 10.1209/epl/i2006-10408-x Henrich M.J., Mahler G., Michel M., Phys. Rev. E, 2007, 75, 051118; doi 10.1103/PhysRevE.75.051118.

10. Allahverdyan A.E., Johal R.S., Mahler G., Phys. Rev. E, 2008, 77, 041118; doi:10.1103/PhysRevE.77.041118

11. Lankaster P., Theory of Matrices. Academic Press, New York-London, 1969.

12. Tapia V. Preprint arXiv:math-ph/0702001v1, 2007.

13. Ivanchenko E.A., J. Math. Phys., 2009, 50, 042704; doi 10.1063/1.3116167.

14. Verstraete F., Dehaene J., De Moor B., Verschelde H., Phys. Rev. A, 2002, 65, 052112; doi 10.1103 /PhysRevA.65.052112

15. Ivanchenko E.A., Low Temp. Phys., 2007. 33, 336; doi 10.1063/1.2720081][Fiz. Nizk. Temp., 2007, 33, 455 (in Russian)].

16. Weimer H., Mahler G., Phys. Rev. A, 2007, 76, 053819; doi 10.1103/PhysRevA.76.053819.

17. Weimer H., Henrich M.J., Rempp F., Schroder H., Mahler G., Europhys. Lett., 2008, 76, 30008; doi: $10.1209 / 0295-5075 / 83 / 30008$

18. Barankov R., Polkovnikov A., 2008; Preprint arXiv:cond-mat/0806.2862, 2008.

19. Schlienz J., Mahler G., Phys. Rev. A, 1995, 52, 4396; doi 10.1103/PhysRevA.52.4396

20. Landau L.D., Phys. Z. Sowjetunion, 1932, 2, 46.

21. Zener C. Proc. R. Soc. London Ser. A, 1932, 137, 696.

22. Stueckelberg E.C.G., Helv. Phys. Acta, 1932, 5, 369.

23. Landau L.D., Lifshitz E.M., Statistical Physics I. Pergamon Press, Oxford, 1978.

24. Callen H.B., Thermodynamics. John Wiley, New York, 1985.

25. Balian R., From Microphysics to Macrophysics, 1, Springer, 1992.

26. Esposito M., Mukamel S., Phys. Rev. E, 2006, 73, 046129; doi:10.1103/PhysRevE.73.046129 Preprint arXiv:cond-mat/0602679v1, 2006.

27. Schrőder H., Teifel J., Mahler G., Eur. Phys. J. Special Topics, 2007, 151, 181; doi:10.1140/epjst/e2007-00373-6.

28. Zhang G-F., Eur. Phys. J. D, 2008, 49, 123; doi 10.1140/epjd/e2008-00133-0 Preprint arXiv:quant$\mathrm{ph} / 0808.2409 \mathrm{v} 1,2008$.

29. Allahverdyan A.E., Balian R., Nieuwenhuizen Th.M., 2004; Preprint arXiv:cond-mat/0401574v1, 2004.

30. Boykin P.O., Mor T., Roychowdhury V., Vatan F., Vrijen R., Proc. Natl. Acad. Sci. USA, 2002, 99, 3388; doi $10.1073 /$ pnas. 241641898 .

31. Quan H.T., Phys. Rev. E, 2008, 79, 041129; doi 10.1103/PhysRevE.79.041129 Preprint arXiv:quant$\mathrm{ph} / 0811.2756 \mathrm{v} 1,2008$ and references therein.

32. He J., Chen J., Hua B., Phys. Rev. E, 2002, 65, 036145; doi 10.1103/PhysRevE.65.036145

33. Linden N., Popescu S., Skrzypczyk P., 2010; Preprint arXiv:quant-ph/0908.2076v2, 2009. 


\section{Нерівноважна оборотна динаміка роботи в 4-спіновій системі у магнітному полі}

\section{Є.О. Іванченко}

Інститут теоретичної фізики, Національний науковий центр “Харківський фізико-технічний інститут”, Харків, Україна

За допомогою декомплексифікації рівняння Ліувіля-Неймана одержана замкнута система рівнянь локальних векторів Блоха та спінових кореляційних функцій для системи 4 магнітних частинок 3 обмінною взаємодією, які знаходяться в довільному залежному від часу магнітному полі. Виконано аналітичний та чисельний аналіз динаміки квантових термодинамічних параметрів в залежності від сепарабельного початкового стану системи та модуляції магнітного поля. В умовах унітарної еволюції досліджено нерівноважну оборотну генерацію роботи у найближчому оточенні.

Ключові слова: оборотна динаміка, генерація роботи, спінова система, магнітне поле 